\title{
Toward Interactive Learning by Concept Ordering
}

\author{
Shantanu Godbole* \\ IBM India Research Lab
}

\author{
Sachindra Joshi \\ IBM India Research Lab \\ Ganesh Ramakrishnan \\ IBM India Research Lab
}

\author{
Sameep Mehta \\ IBM India Research Lab
}

\begin{abstract}
In this paper we present a visual education tool for efficient and effective learning. The toolkit is based on a simple premise: simple concepts should be learned before advanced ones. We propose algorithms to automatically capture such pre-requisite dependence relationships between concepts. We extract concept definitions from the web's hyperlinked environment and create a concept graph arranged in a hierarchical structure and presented to the user in an interactive fashion. Thereafter, the user guides the learning process in a hyperlinked environment, by selecting a target concept, exploring the associated learning graph, learning pre-requisite concepts, and repeating this process till her learning goal is reached. To measure usefulness and correctness of our approach, we conducted a user study with 25 users using precision and recall measures. Overall, the feedback from users was encouraging. We believe this is a positive step toward building user driven interactive learning systems.
\end{abstract}

\section{SYSTEM DESCRIPTION}

1.1 Motivation: Traditionally, individuals have relied on books, newspapers and other printed material to learn. However, recent past has seen a radical shift in the learning paradigm with the advent of the Internet. In the presence of efficient and accurate search engines, a user searches a target concept on the web and learns it by following relevant (typically top 10) results. This method indeed looks attractive but it unfortunately lacks the structure provided by printed sources. For example, concepts in printed books are arranged in increasing order of difficulty and maturity. Any such ordering is missing from the Search-and-Learn paradigm. While reading about a concept using online resources, the user has to follow hyperlinks and navigate back and forth between related, underlying or pre-requisite concepts. This navigation is ad-hoc without any principled ordering. However, online learning supports very effective

\footnotetext{
*\{shgodbol,jsachind,sameepmehta,ganramkr\}@in.ibm.com
}

Permission to make digital or hard copies of all or part of this work for personal or classroom use is granted without fee provided that copies are not made or distributed for profit or commercial advantage and that copies bear this notice and the full citation on the first page. To copy otherwise, to republish, to post on servers or to redistribute to lists, requires prior specific permission and/or a fee.

Hypertext '07 Manchester, United Kingdom

Copyright 200X ACM X-XXXXX-XX-X/XX/XX ...\$5.00. browsing between concepts which is not possible in books. In this paper, we develop a visual educational tool which aims to leverage the positive features of online and print-based approaches while mitigating their negative aspects and helps the user learn effectively and efficiently.

The tool is based on simple observations about learning: i)learning any (fairly) advanced concept involves learning pre-requisite concepts and ii) outside of the classroom settings, individuals prefer focused learning and would not like to spend time on already known (redundant) or peripheral (non-relevant) concepts. We contend that both these criteria can be satisfied by arranging the concepts in a hierarchical graph-like structure. To be more precise, there is a partial ordering between concepts, which corresponds to a directed acyclic graph (DAG). We employ graph theoretic algorithms to construct these concept graphs automatically, mining and extracting them from the web. The concept graphs are presented to the user in an interactive environment, where the user can see all the relevant concepts and can choose to explore non-redundant (previously unknown) concepts in detail, while ignoring known concepts.

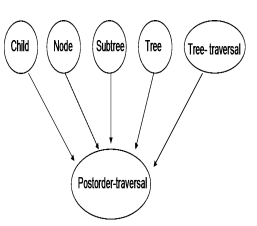

(a)

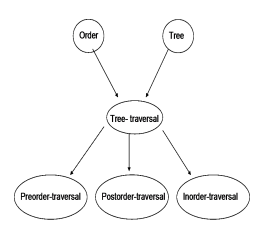

(b)

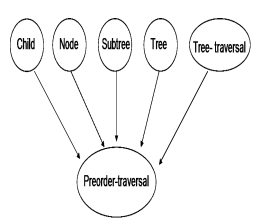

(c)
Figure 1: Example showing the concept graphs for types of tree traversal

Before providing the brief summary of the algorithms, we present a concrete example. Assume that the user wants to learn post-order traversal. The corresponding learning graph is shown in Figure 1(a). However, by inspecting the graph, the user soon realizes that she needs to understand tree traversal. She clicks on the tree traversal node and the learning graph shown in Figure 1(b) is presented. The user learns tree-traversal and realizes that she can also learn preorder and in-order traversal without learning any new concepts. Figure 1(c) is displayed when she selects pre-order traversal. This expository example illustrates the usefulness of our system, wherein the user needs an interactive environment to learn the target concept (post-order traversal) and at the same time also realizes that extra concepts can be learned without much effort. 


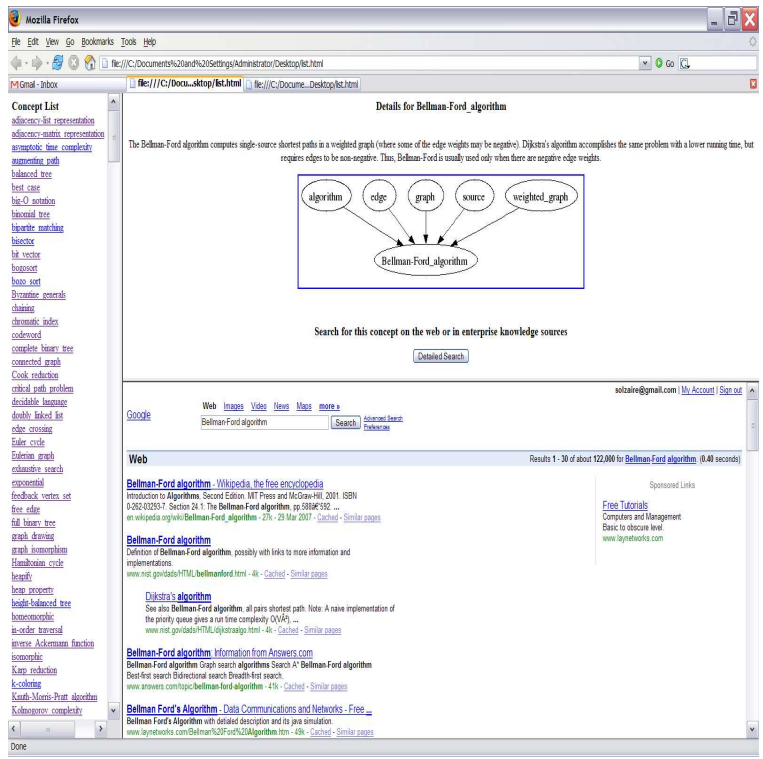

Figure 2: Snapshot of the web based visual tool

1.2 Algorithm: Let $C$ be a list of $N$ concepts, and let $C[i]$ refer to the the $i^{\text {th }}$ concept in the list. We assume the availability of a database of concept definitions for a given domain ${ }^{1}$. Using this definition database, we construct the concept graph $C_{D A G}$ for a particular domain. Our algorithm for constructing the concept DAG consists of two steps. In our first step, we construct a graph $C_{\text {graph }}$ by making use of references between concepts in concept definitions. In the second step, we extract a concept DAG $C_{D A G}$ from $C_{g r a p h}$. Step 1: We generate a weighted and directed concept graph $C_{\text {graph }}=(V, E)$ as follows. For every concept $C[i], 1 \leq i \leq$ $N$, we create a vertex $v_{i}$ in $V$. An edge is introduced from $v_{i}$ to $v_{j}$ iff concept $C[j]$ is mentioned in some definition of $C[i]$. We say that 'concept $C_{1}$ is mentioned in some text' iff the lemma form of $C_{1}$ matches the lemma form of some token in the text. The weight $w_{i j}$ of an edge $\left(v_{i}, v_{j}\right)$ is set to the number of mentions of $C[j]$ in the definitions of $C[i]$. Step 2: Extracting a DAG $C_{D A G}$ from $C_{\text {graph }}$ requires the elimination of cycles from $C_{\text {graph }}$. There could be several sets of edges whose removal would lead to elimination of cycles. In our case of a weighted digraph, it makes sense to remove the set of edges with minimum total weight. The problem of removing minimum number of edges in a digraph is a standard problem studied in literature as the minimum feedback arc-set problem (FAS) [4]. Unfortunately, the problem is NP-Hard [3]. However, due to the wide applicability of the FAS problem, several heuristics have been proposed for it. For our task, we use a variant of a fast, effective and simple heuristic for FAS proposed by Eades, et. al. [1].

1.3 Interactive Visual Interface: Next, we outline the key components of our web-based interface. A snapshot of the interface is shown in figure 2. A concept list is the list of all concepts in a domain of interest. The list is displayed in the left pane of our tool. The user can select the concept of interest from the list and its associated learning graph is

${ }^{1}$ We used Google's "define:" query operator [2] to build the database given an index of concepts on the web displayed on the right. Learning Graph shows the concept selected from the concept list and it's pre-requisite nodes. In addition to these ancestor nodes, we also show one level of descendants from our concept index. These are nodes which need the target concept as their pre-requisite. The learning graph is shown in top pane. The nodes of the graph are clickable and can also be used to select the target concept. The process is the same as choosing a concept from the concept list. Concept Definitions are the actual extracted definitions of the concept shown above the graph. The bottom pane shows the concept's web query search results.

1.4 Overall Process: The concept list is obtained from either online glossaries or indexes present at the back of books. Corresponding concept definition database is built. The list and database are used to derive the concept DAG. This process is executed offline. The list is populated in the visual interface. The user selects a concept from the concept index and the associated learning graph is presented. Next, the user identifies the pre-requisite concept to be learned and clicks on the corresponding node. A new learning graph is displayed in real time. In order to maintain the context sensitivity of the learning graph, the parent node (original target to be learned) is always displayed. In our user study we found that this property helps the user stay focused and reduces navigational time. Once the user has learned all pre-requisites, she reads the definition of the target concept. Subsequently, based on her interest, the user may search other data sources and refine or broaden her understanding.

1.5 Experiment: We designed and conducted a user study with 20 participants to evaluate the quality of our concept graphs. Every user was presented with learning graphs corresponding to 15 concepts and asked to mark redundant (false positive) and missing edges (false negatives). These numbers are used to calculate precision and recall. The average precision and recall were found to be .80 ( standard deviation of .16) and .84 (.12) respectively.

1.6 Conclusions: In this paper, we presented an interactive visual toolkit to facilitate the learning process by capturing the pre-requisite dependences between concepts in learning domains. These dependences are modeled hierarchically and presented to the user. She can recursively learn pre-requisite concepts to thoroughly understand target concepts of interest according to learning goals. We evaluated our approach by conducting a moderately large user study using the widely accepted precision and recall metrics to measure correctness and usefulness respectively. Overall, the users found the toolkit to be very useful for learning concepts in a principled and organized fashion. Currently, we are exploring algorithms to incorporate each user's existing skill-set to set up a domain specific baseline, which will further result in focused learning and reduce learning time.

\section{REFERENCES}

[1] Peter Eades, Xuemin Lin, and W. F. Smyth. A fast and effective heuristic for the feedback arc set problem. Information Processing Letters, 47(6):319-323, 1993.

[2] Google. Advanced google search operators, 2002.

[3] R. Karp. Reducibility among combinatorial problems. In Complexity of Computer Communications, pages 85-103, 1972.

[4] P. Slater. Inconsistencies in a schedule of paired comparisons. Biometrika, 48:303-312, 1961. 